
2 Research Square
Preprints are preliminary reports that have not undergone peer review.
They should not be considered conclusive, used to inform clinical practice, or referenced by the media as validated information.

\title{
Correlation of Small Bowel Gastrointestinal Stromal Tumor Computed Tomography Features with the Grade of Pathological Risk
}

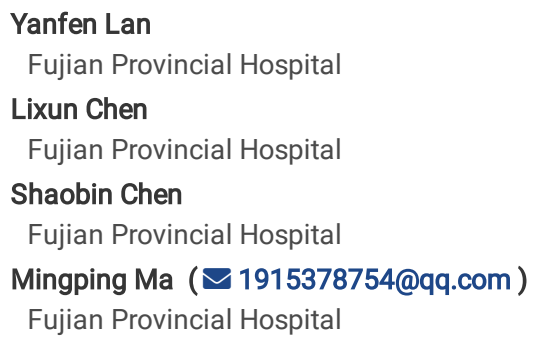

Research Article

Keywords: small bowel gastrointestinal stromal tumors, CT imaging performance, risk gradation, operation, postoperative pathology

Posted Date: October 11th, 2021

DOI: https://doi.org/10.21203/rs.3.rs-934053/v1

License: (9) (1) This work is licensed under a Creative Commons Attribution 4.0 International License. Read Full License 


\section{Abstract}

Objectives: The aim of this study was to investigate the diagnostic value of computerized tomography (CT) features of small intestinal stromal tumors in terms of their degree of risk.

Methods: The clinical data and CT data of 107 patients with small intestinal stromal tumors confirmed by surgery and pathology in our hospital from June 2012 to October 2020 were selected. According to the results of postoperative pathological risk, the patients were divided into high-risk and low-risk groups, wherein 67 cases were in high-risk group and 40 cases were in the low-risk group The maximum diameter, solid component plain scan, arterial phase CT value, venous phase CT value, and delayed phase CT value of the two groups were measured, and the enhancement degree of arterial phase, venous phase, delayed CT value, and lesion enhancement mode were calculated. The difference between the two groups was compared. An independent sample t-test was used to compare quantitative indices, and the chi-squared test or Fisher's exact test was used for qualitative index comparison. A receiver operating characteristic (ROC) curve was used to evaluate the diagnostic value of the arterial phase CT value, venous phase CT value, delayed phase CT value, arterial phase enhancement degree, venous phase enhancement degree, delayed phase enhancement degree, and the enhanced net value-added in the risk degree of SBGISTs. The relationship between preoperative imaging findings and tumor risk was retrospectively analyzed.

Results: Univariate analysis showed that there were significant differences in the lesion location, growth pattern, lesion ulcer, necrotic cystic degeneration, lobulation, boundary with surrounding tissues, plain scan density and lesion enhancement mode, CT value in arterial phase, increment in arterial phase, CT value in venous phase, increment in venous phase, CT value in delayed phase, increment in delayed phase, and enhancement value in lesion between the two groups $(\mathrm{P}<0.05)$; there were no significant differences in sex, age, calcification, bleeding, clinical symptoms, and CT value $(P>0.05)$. The ROC curve analysis showed that the area under the curve (AUC) of the long diameter of the lesion was $0.959(P=0.000)$, the optimal critical point of the ROC curve was the lesion $\geq 4.80 \mathrm{~cm}$, the sensitivity was $88.1 \%$, the specificity was $97.5 \%$, and the accuracy was $91.6 \%$; for the low-risk group, the AUC was 0.788 (the largest, $P=0.000$ ), the sensitivity was $77.5 \%$, the specificity was $70.1 \%$, and the accuracy was $72.9 \%$. Multivariate analysis showed that non-uniform density $(P=0.030 ;$ odds ratio [OR]: 12.544; 95\% confidence interval [CI]: 1.269-123.969), arterial phase $C T$ value $(P=0.024 ; 0 R: 10.790 ; 95 \% \mathrm{Cl}: 1.374-84.754)$, and lesion length $(P=$ 0.000; OR: 648.694; 95\% Cl: 40.541-10,379.714) were risk factors for SBGISTs.

Conclusions: The CT features of small intestinal stromal tumors have certain characteristics, which can help to grade the risk of small intestinal stromal tumors before surgery.

\section{Introduction}

Small bowel gastrointestinal stromal tumors (SBGISTs), a type of gastrointestinal stromal tumors (GISTs), are mesenchymal tumors accounting for approximately $20 \%$ of GISTs and $8 \%-15 \%$ of primary small intestinal tumors ${ }^{[1]}$. Patients mostly present with abdominal pain, gastrointestinal bleeding, or a palpable abdominal mass ${ }^{[2,3]}$. The blood supply of small intestinal stromal tumors is high. The treatment of SBGISTs varies, depending on the different locations, sizes, and risk degrees ${ }^{[4-7]}$.Preoperative CT multi-directional analysis can determine the origin of the lesion, and imaging characteristics can assist the clinical diagnosis and treatment. In this study, we aimed to investigate the diagnostic value of computerized tomography (CT) features of small intestinal stromal tumors in terms of their degree of risk.

\section{Methods Patients}

One-hundred seven patients with SBGISTs that were confirmed by operation and pathological examinations in our hospital from June 2012 to October 2020 were retrospectively included in the study, particularly, 54 men and 53 women, aged 30-78 years, with an average age of $57.60 \pm 11.07$ years. The main clinical symptoms were black or bloody stool, abdominal pain, abdominal distension, abdominal mass, one case of gastrointestinal perforation, 51 cases of gastrointestinal bleeding, and 55 cases of bleeding in 55 cases, wherein 31 cases were found by physical examination. All patients underwent multi-slice CT plain scans and three-phase enhanced scans before surgery, including 78 cases using Siemens Sensation 64 spiral CT ( Siemens, Germany), seven cases using Siemens SOMATOM force spiral CT ( Siemens, Germany), and 15 cases using Siemens SOMATOM definition spiral CT ( Siemens, Germany).

\section{Inclusion and exclusion criteria}

Eleven patients who had primary single SBGISTs and underwent plain CT and three-phase enhanced CT were included in the study. On the other hand, patients with liver metastasis, intraperitoneal implantation metastasis, and other metastases; patients with acute abdomen such as gastrointestinal perforation, previous tumor history, or other abdominal lesions; and patients who underwent reoperation after chemotherapy and reoperation after recurrence were excluded from the study.

The pathological risk grading criteria and grouping were mainly based on the published guidelines, "Chinese consensus guidelines for diagnosis and management of gastrointestinal stromal tumors (2017 Edition)," to grade the risk of SBGISTs. ${ }^{[7]}[$

Routine bowel preparation was performed before the examination. First, 1,500-2,000 mL of 2\% isotonic mannitol solution (180719B24, Fuzhou Haiwangfuyao Pharmaceutical Co., Ltd., Chengdou, China) was taken orally by stages within 60-90 min before the examination, and then 20-mg drotaverine hydrochloride injection (7CE07A, Sanofi [Hangzhou] Pharmaceutical Co., Ltd., Shanghai, China) was given intramuscularly 15-20 min before the examination. The patient was placed in the supine position for anal intubation. Then, $800-1,000 \mathrm{~mL}$ of $2 \%$ isotonic mannitol solution was infused through the anal canal to 
fill the colon. Multislice spiral CT (MSCT) scanning and Post-Processing Workstation were used. The scanning parameters were $120 \mathrm{kV}, 200-350 \mathrm{~mA}$, tube speed of $0.5 \mathrm{~s} \mathrm{cycle}^{-1}$, slice thickness of $3 \mathrm{~mm}$, pitch of 1.2 , bed speed of $27.5 \mathrm{~mm}$ cycle ${ }^{-1}$, standard reconstruction algorithm reconstruction, and volume scanning from the diaphragmatic apex to the pubic symphysis in the breath-holding state. The patients were scanned at the arterial, venous, and delayed phases at $25 \mathrm{~s}, 50-70 \mathrm{~s}$, and $300 \mathrm{~s}$ after intravenous injection of $80-100 \mathrm{~mL}$ of the iodine contrast agent loversol $\left(320 \mathrm{mgl} \mathrm{mL}^{-1}\right.$; Jiangsu Hengrui Pharmaceutical Co., Ltd.) with a flow rate of 3-4 mL. Data on the 1.0-mm thick samples for the four-phase scanning were transferred to the Workstation (WWMP or syngo.via)( Siemens, Germany) for processing and analysis.

The CT images were independently completed by two doctors with more than 5 years of experience in the imaging diagnosis of digestive system diseases. The double-blind method was used for the diagnosis. Consensus was reached through consultation when opinions were different. In addition to the intraoperative data, the location, size, scope, shape, boundary of the tumors; whether the tumor was combined with necrotic cystic change, ulcer, bleeding; calcification, plain scan density, and uniformity; CT value and maximum CT value of each phase after enhancement; and enhancement mode, structural changes around the lesion, lymph node, or abdominal cavity metastasis were evaluated. Lesion density was based on the muscles of the same layer.

For the CT value measurement, the region of interest (ROI) was placed in the solid part of the lesion and the most obvious part of enhancement, avoiding blood vessels, calcification, necrosis, and edge. The ROls in the different stages were placed at the same level, using the same size and shape. For the enhanced CT measurement, the ROI was placed at the same level in each phase, and the CT increment was calculated using the following:

$$
\begin{gathered}
\text { CTincrement }=\text { CTvalueofeachphase }- \text { plainCTvalue } \\
\text { netincrement }=\text { highestenhancedCTvalue }- \text { plainCTvalue }
\end{gathered}
$$

There are three ways of enhancement: (1) fast-in and slow-out, which is the most obvious enhancement in the arterial phase and gradually decreases in the venous phase and delayed phase; (2) gradual slow-out, which is the most obvious enhancement in the venous phase and slightly decreases in the delayed phase; and (3) gradual enhancement, which is the gradual enhancement from the arterial phase to the delayed phase and is the most obvious enhancement in the delayed phase. The two doctors were trained to judge uniformity, which was determined as uniform or uneven. Gastrointestinal bleeding is defined as the presence of black or bloody stools.

Statistical analysis was performed using SPSS 25.0. For continuous measurement data, in the form of mean \pm standard deviation, an independent sample ttest was used for comparison of the mean values between groups. Counting data were expressed as rate, and the chi-square test was used. In order to exclude the influence of confounding factors, binary logistic regression analysis was performed, and $P<0.05$ was considered statistically significant. The area under the curve (AUC), sensitivity, and specificity were then calculated.

\section{Results}

There were 107 cases, 67 cases (71.9\%) with high risk of SBGISTs, no cases with medium risk, 34 cases with low risk, and six cases with extremely low risk. Given that there were no medium-risk cases and only six cases had extremely low risk, there was no statistical significance. Therefore, the extremely low-risk and low-risk patients were included in the low-risk group, while the high-risk patients were included in the high-risk group. There was no significant difference in clinical characteristics between the two groups (Table 1).

Univariate analysis on the relationship between preoperative CT signs and the risk of lesions showed that the imaging manifestations, including the boundary between the lesion and surrounding tissue, ulcer, necrotic cystic change, lesion lobulation, plain scanning density, lesion location, growth mode, enhancement mode, arterial phase CT value, arterial phase increment CT value, venous phase CT value, venous phase increment CT value, delayed phase CT value, delayed phase increment CT value, enhanced net value, and lesion length, were significantly associated with the risk of SBGISTs between the two groups $(P<0.05)$, as shown in Tables 2 and 3.

For the receiver operating characteristic (ROC) curve analysis of the high-risk group, the AUC of the lesion length and diameter was $0.959(P=0.000)$, and the best critical point of the ROC curve was a lesion $\geq 4.80 \mathrm{~cm}$, with a sensitivity of $88.1 \%$, specificity of $97.5 \%$, and accuracy of $91.6 \%$. The ROC curve analysis of the low-risk group is shown in Table 4 and Figures 3 and 4.

According to ROC curve analysis, the critical values of the arterial phase CT values, arterial phase enhancement values, venous phase CT values, venous phase enhancement values, delayed phase CT values, delayed phase enhancement values, and net enhancement values were calculated and divided into groups. These data were included in the multivariate logistic regression analysis. The results showed that the non-uniform density $(P=0.034 ;$ odds ratio [OR]: 12.544; 95\% confidence interval [Cl]: 1.269-123.969), arterial phase CT value ( $P=0.024 ;$ OR: $10.790 ; 95 \%$ Cl: $1.374-84.754)$, and lesion length $(P=0.000 ;$ OR: 648.694; 95\% Cl: 40.541-10,379.714) were risk factors for SBGISTs, as shown in Table 5. 
Table 1

Comparison of clinical characteristics ofSB GISTsbetween the two groups

\begin{tabular}{|lllllll|}
\hline group & \multirow{2}{*}{ Age(year, $\mathbf{X} \pm \mathbf{s})$} & \multicolumn{2}{l}{ Gender } & \multicolumn{2}{l|}{ Gastrointestinal bleeding } \\
\cline { 4 - 7 } & & & male & female & Yes & No \\
\hline Low risk group & 40 & $57.28 \pm 9.884$ & 21 & 19 & 20 & 20 \\
\hline High risk group & 67 & $57.79 \pm 11.79$ & 33 & 34 & 32 & 35 \\
\hline Statistics & $-0.232^{\mathrm{b}}$ & $0.106^{\mathrm{a}}$ & $0.823^{\mathrm{a}}$ & \\
\hline Pvalue & 0.817 & 0.745 & 0.050 & \\
\hline Note: a: $\chi^{2}$ value;b:t value & & & & \\
\hline
\end{tabular}

Table 2

Single factor analysis results of CT signs and postoperative risk ofSB GISTsin two groups

\begin{tabular}{|c|c|c|c|c|c|c|c|c|c|c|c|c|c|}
\hline \multirow[t]{2}{*}{ group } & \multirow[t]{2}{*}{$\mathbf{n}$} & \multicolumn{3}{|l|}{ Lesion location } & \multirow{2}{*}{\multicolumn{2}{|c|}{$\begin{array}{l}\text { Diameter of } \\
\text { lesion }(\mathrm{cm}, \mathbb{\nabla x} \pm \mathrm{s})\end{array}$}} & \multicolumn{5}{|c|}{ Growth pattern } & \multicolumn{2}{|c|}{ boundary } \\
\hline & & duodenum & jejunum & ileum & & & \multicolumn{2}{|c|}{ intracavity } & \multicolumn{2}{|c|}{ Intraluminal } & Extraluminal & Clear & Unclear \\
\hline $\begin{array}{l}\text { Low risk } \\
\text { group }\end{array}$ & 40 & 17 & 29 & 3 & \multicolumn{2}{|c|}{$3.150 \pm 1.039$} & \multicolumn{2}{|l|}{6} & \multicolumn{2}{|l|}{19} & 15 & 34 & 6 \\
\hline $\begin{array}{l}\text { High risk } \\
\text { group }\end{array}$ & 67 & 14 & 33 & 20 & \multicolumn{2}{|c|}{$7.812 \pm 3.148$} & \multicolumn{2}{|l|}{2} & \multicolumn{2}{|l|}{27} & 38 & 27 & 40 \\
\hline Statistics & & \multicolumn{3}{|l|}{$9.859^{a}$} & \multicolumn{2}{|c|}{$-11.15^{b}$} & \multicolumn{4}{|c|}{$7.005^{\mathrm{a}}$} & & \multicolumn{2}{|l|}{$20.42^{a}$} \\
\hline$P$ value & & \multicolumn{3}{|l|}{0.007} & \multicolumn{2}{|c|}{0.000} & \multicolumn{4}{|c|}{0.030} & & \multicolumn{2}{|l|}{0.000} \\
\hline \multirow[t]{2}{*}{ group } & \multicolumn{2}{|l|}{ Plain density } & \multicolumn{2}{|l|}{ ulcer } & \multicolumn{2}{|c|}{$\begin{array}{l}\text { Necrotic cystic } \\
\text { degeneration }\end{array}$} & \multicolumn{2}{|c|}{ hemorrhage } & \multicolumn{2}{|c|}{ calcification } & \multicolumn{3}{|c|}{ enhancement pattern } \\
\hline & homogeneous & heterogeneous & No & Yes & No & Yes & No & Yes & No & Yes & $\begin{array}{l}\text { fast in and } \\
\text { slow out }\end{array}$ & $\begin{array}{l}\text { gradual } \\
\text { slow } \\
\text { out }\end{array}$ & $\begin{array}{l}\text { gradual } \\
\text { enhancer }\end{array}$ \\
\hline $\begin{array}{l}\text { Low risk } \\
\text { group }\end{array}$ & 25 & 15 & 33 & 7 & 31 & 9 & 39 & 1 & 35 & 5 & 17 & 17 & 6 \\
\hline $\begin{array}{l}\text { High risk } \\
\text { group }\end{array}$ & 17 & 50 & 35 & 32 & 35 & 32 & 59 & 8 & 52 & 15 & 19 & 16 & 32 \\
\hline Statistics & $14.48^{\mathrm{a}}$ & & $9.902^{\mathrm{a}}$ & & 6.762 & & 2.89 & & 1.61 & & $11.87^{\mathrm{a}}$ & & \\
\hline$P$ value & 0.000 & & 0.002 & & 0.009 & & 0.08 & & 0.20 & & 0.003 & & \\
\hline
\end{tabular}

Note: a: $\chi^{2}$ value;b:t value

Table 3

Single factor analysis results of CT enhancement characteristics and postoperative risk between the two groups

\begin{tabular}{|c|c|c|c|c|c|c|c|c|c|}
\hline \multirow[t]{2}{*}{ group } & \multirow[t]{2}{*}{$\mathbf{n}$} & \multicolumn{8}{|c|}{ Lesion component density (HU) } \\
\hline & & plain & $\begin{array}{l}\text { arterial } \\
\text { phase }\end{array}$ & $\begin{array}{l}\text { arterial phase } \\
\text { increment }\end{array}$ & $\begin{array}{l}\text { venous } \\
\text { phase }\end{array}$ & $\begin{array}{l}\text { venous phase } \\
\text { increment }\end{array}$ & $\begin{array}{l}\text { delayed } \\
\text { phase }\end{array}$ & $\begin{array}{l}\text { delayed phase } \\
\text { increment }\end{array}$ & $\begin{array}{l}\text { enhanced } \\
\text { net }\end{array}$ \\
\hline $\begin{array}{l}\text { Low risk } \\
\text { group }\end{array}$ & 40 & $36.06 \pm 6.061$ & $111.7 \pm 38.59$ & $75.59 \pm 37.10$ & $117.2 \pm 31.09$ & $81.14 \pm 30.60$ & $87.46 \pm 17.95$ & $51.40 \pm 17.11$ & $87.79 \pm 34.09$ \\
\hline $\begin{array}{l}\text { High risk } \\
\text { group }\end{array}$ & 67 & $36.58 \pm 5.370$ & $76.77 \pm 30.05$ & $40.19 \pm 29.06$ & $90.12 \pm 31.60$ & $53.54 \pm 30.65$ & $76.41 \pm 18.27$ & $39.83 \pm 17.48$ & $56.39 \pm 29.64$ \\
\hline Statistics & & -0.232 & 4.899 & 5.488 & 4.315 & 4.509 & 3.046 & 3.337 & 5.010 \\
\hline$P$ value & & 0.817 & 0.000 & 0.000 & 0.000 & 0.000 & 0.003 & 0.001 & 0.000 \\
\hline
\end{tabular}


Table 4

Area analysis under ROC curve

\begin{tabular}{|lllllll|}
\hline Variable & AUC & Pvalue & $95 \% \mathrm{Cl}$ & Sensitivity(\%) & Specificity(\%) & Accuracy(\%) \\
\hline Lesion length(cm) & 0.959 & 0.000 & $0.924-0.993$ & 88.1 & 97.5 & 91.6 \\
\hline Arterial phase(HU) & 0.775 & 0.000 & $0.686-0.864$ & 87.5 & 58.2 & 69.2 \\
\hline Arterial phase Increment(HU) & 0.788 & 0.000 & $0.700-0.877$ & 77.5 & 70.1 & 72.9 \\
\hline Venous phase(HU) & 0.737 & 0.000 & $0.642-0.833$ & 67.5 & 76.1 & 72.9 \\
\hline Venous phase Increment(HU) & 0.747 & 0.000 & $0.653-0.841$ & 67.5 & 76.1 & 72.9 \\
\hline Delayed phase(HU) & 0.675 & 0.003 & $0.570-0.780$ & 87.5 & 43.3 & 59.8 \\
\hline Delayed phase Increment(HU) & 0.693 & 0.001 & $0.591-0.795$ & 82.5 & 52.2 & 63.6 \\
\hline Enhanced net(HU) & 0.766 & 0.000 & $0.674-0.858$ & 70 & 76.1 & 73.8 \\
\hline
\end{tabular}

Table 5

Multivariate analysis of CT signs and pathological risk grade

\begin{tabular}{|llll|}
\hline \multicolumn{4}{c}{ in two groups } \\
\hline variable & OR value & $\mathbf{9 5 \%} \mathbf{C l}$ & $\boldsymbol{P}$ value \\
\hline Scan density & 12.544 & $1.269-123.969$ & 0.030 \\
\hline Arterial phase & 10.790 & $1.374-84.754$ & 0.024 \\
\hline Lesion length & 658.694 & $40.541-10379.714$ & 0.000 \\
\hline
\end{tabular}

MSCT scan showed slightly lower and equal density in transverse section (A), obvious enhancement in arterial phase (B), continuous enhancement in venous phase (C), and decreased enhancement in delayed scanning (D). In coronal position (E), the lesion was located in duodenal cavity, with clear boundary and uniform density. The pathological risk grade was low (F).

MSCT showed a slightly lower density in the horizontal section (A) of plain scan, and patchy necrotic areas were seen in it. In the arterial phase (B) of enhanced scan, the lesions showed uneven and obvious enhancement, with multiple small vessel branches supplying blood in it, no enhancement in the necrotic cystic area, slightly weakened enhancement in venous phase (C) and delayed phase (D), and mesenteric artery branches supplying blood in coronary position (E), The pathological risk level was high (F).

\section{Discussion}

Gastrointestinal stromal tumors are common mesenchymal tumors of the gastrointestinal tract and GISTs are mainly distributed in the stomach, small intestine, colon, and outside the gastrointestinal tract. Distribution in the small intestine accounts for approximately $20 \%$ of the total cases of GISTs. Malignant stromal tumors mainly metastasize to the liver, peritoneum, lung, bone, lymph node, and skin, and subcutaneous metastases are relatively rare. Metastases to the bone head are high-risk stromal tumors. ${ }^{\left[{ }^{8-10]}\right.}$ Gastrointestinal stromal tumors mainly occur in patients over 50 years of age, with a median age of 55-65 years old, but there is no gender preference. The incidence is $5-20 \%$ in patients younger than 40 years of age, while incidence in children is rare. ${ }^{[9]}$ In this study, the age of the patients was in the range of 30-78 years old, with a mean of $57.60 \pm 11.07$ years old. There was no significant difference in gender and age between the two groups (Table 1), which is consistent with previous reports. The common clinical symptoms of SBGISTs are gastrointestinal bleeding, abdominal pain, abdominal mass, complete or incomplete intestinal obstruction, and occasional gastrointestinal perforation, and some of the clinical symptoms are not obvious, similar to the clinical symptoms of other small intestinal tumors. Moreover, there is no obvious special performance and is more prone to misdiagnosis ${ }^{[11,12]}$ There was no significant difference in gastrointestinal bleeding between the two groups $(P>0.05)$. According to the age and gender of patients, clinical symptoms, and risk of SBGISTs, we should be attentive for the possible diagnosis of SBGISTs when reading the film and try to reduce misdiagnosis and missed diagnosis. Eighty percent of GIST cases are associated with tyrosine kinase protein (c-KIT) mutation. Moreover, immunohistochemical analysis of GISTs usually show the expression cluster of differentiation 117 (CD117) and discovered on gastrointestinal stromal tumors protein 1 (DOG1). Positive CD117 expression can distinguish SBGISTs from small intestinal tumors. ${ }^{[13-15]}$ The results for CD117, DOG1, and cluster of differentiation 34 (CD34) expression in this study were $100 \%, 100 \%$, and $90.7 \%$, respectively, which are consistent with those reported in the literature.

Univariate analysis of SBGISTs showed that there were significant differences between the low-risk and high-risk groups in terms of lesion length, boundary between the lesion and surrounding tissue, lesion lobulation, unenhanced density, ulcer, necrotic cystic degeneration, growth pattern, and lesion location (Table 2,3). Multivariate analysis identified uneven tumor unenhanced density as an independent risk factor for high-risk SBGISTs (Table 5). We believe that when the growth of tumor cells is more active, the growth rate of small blood vessels in the tumor is less than that of tumor cells, and the blood supply is insufficient, which leads to uneven density CT manifestations such as ulcers, necrotic cysts, and bleeding. It has been reported that the CT signs of gastrointestinal GISTs such as tumor edge, shape, tumor growth pattern, necrosis, and enhancement pattern are related to risk stratification ${ }^{[16-19]}$. Mazzei MA et al ${ }^{[20]}$ show that the CT percentage of intralesional hypodensity as a CT surrogate of mitotic count demonstrates a good correlation with Miettinen risk assessment index in risk

Page 5/10 
stratification of GIST patients, moreover CT percentage value of hypodensity $>20 \%$ can be use as a possible accurate CT index to predict the metastatic potential of GISTs. Huh et al ${ }^{[17]}$ found that gastric GIST tumors combined with necrosis are an independent risk factor. At present, there are no consistent results regarding the risk of bleeding and calcification in SBGISTs. In our study, there was no significant difference between the two groups in bleeding and calcification, which needs to be further explored.

The results of this study showed that tumor length $\geq 4.8 \mathrm{~cm}$ was an independent risk factor for high-risk SBGISTs, which had high accuracy in the diagnosis of high-risk SBGISTs(Table 5). This indicated that in SBGISTs, a tumor with a maximum diameter greater than $4.8 \mathrm{~cm}$ can be considered high-risk SBGISTs. At present, many studies have found that tumor length $\geq 5 \mathrm{~cm}$ is an independent risk factor for gastrointestinal GISTs ${ }^{[10,14,18,19,21]}$, which is similar to the results of this study. Our results showed that there was a significant difference in the growth pattern of tumors between the two groups, which indicated that the risk of SBGISTs in different growth patterns was different. There were no significant results from the multivariate analysis. At present, studies have found that extraluminal tumor growth is an independent risk factor for gastric GISTs. ${ }^{[18][19]}$ Our results showed that 38 cases of extraluminal growth were the most common in the high-risk group, and the tumors were all large. The growth pattern of tumors had reference significance for the preoperative risk grading of SBGISTs. In our study, the difference between the duodenum, jejunum, and ileum between the two groups was statistically significant. The incidence rate of SBGIST in the jejunum was higher than those of the duodenum and ileum. The results of the multivariate analysis were not statistically significant. We found that only three cases SBGIST in the ileum were found in the low-risk group, which may be related to the small sample size. We also found that lesion location was an independent risk factor for gastrointestinal GISTs. The risk of non-gastric GISTs is higher than that of gastric GISTs. ${ }^{[13,14]}$ Currently, there is limited research on the SBGIST fraction. Studies have suggested a higher incidence in the jejunum than in the ileum; however, the risk level was not analyzed. ${ }^{[10][22]}$

The results of this study found that the CT values in all phases of CT enhancement, the added value of contrast-enhanced CT, and the enhancement modality differed significantly between the two groups. The ROC curve analysis of arterial phase CT values, arterial phase added values, venous phase added values, and the net value of enhancement in the venous phase showed some accuracy for the diagnosis of SBGISTs with lower risk, CT values in the arterial phase and the degree of enhancement in the arterial phase were more accurate than the other signs(Table 4). The results of the multivariate analysis suggested that the CT value in the arterial phase was an independent risk factor for the risk degree of SBGISTs, and when the CT value in the arterial phase was lower than $72.6 \mathrm{Hu}$, it was suggestive of high-risk SBGISTs. Qian su's et al. found that the CT values in the venous and delayed phases were significantly different between the groups without risk grade SBGISTs. ${ }^{[23]}$ It was found that the blood supply of SBGISTs was abundant, and thickening of the feeding arteries could be seen in the tumor growth sites (Figure 1). The lesion was smaller, and the enhancement was more obvious in the arterial phase than that in the other phases; therefore, the higher the intensity CT value in the arterial phase, the more homogeneous the enhancement was, suggesting a lower degree of danger, as shown in (Figure 2). When the tumor was larger, its enhancement time was relatively prolonged, and the enhancement in the arterial phase was weaker, with gradually slower enhancement or progressive enhancement. The plain CT value results were not statistically different between the two groups, considering that the measured values were all taken from the solid component, so there was no differential result.

\section{limitations}

This study has some limitations. First, we did not collect cases in the intermediate-risk group. We consulted experts in the Department of Pathology of our hospital to check again. Consulting the guidelines, we found that the number of cases in the intermediate-risk group was small, and there was no evidencebased medicine. Reviewing relevant reports at home and abroad, only a few articles have mentioned cases of SBGISTs in the intermediate-risk group. ${ }^{\text {[21, 24] }}$ After discussing and studying with pathology experts, we found that the clinical symptoms of SBGISTs were not typical. Most of the patients with symptoms were found to have larger lesions or higher risk, while patients with smaller lesions were found by physical examination. According to the guidelines, the lesions in the medium-risk group and the very low-risk group were small $(\leq 2 \mathrm{~cm})$, but the mitotic images in the medium-risk group were high, and only six cases in this group were $\leq 2 \mathrm{~cm}$. Moreover, the mitotic images were low, so they were classified into the very low-risk group. The clinical symptoms of patients with small lesions are not obvious, they choose not to operate temporarily, or there is a risk of missed diagnosis due to the small lesions, so it is difficult to collect cases in the medium-risk group. Second, this was a retrospective study that used different CT models and unavoidable selection bias. Therefore, further prospective and multicenter studies with larger sample sizes are required.

\section{Conclusion}

The CT examination and diagnosis of SBGISTs are highly accurate. Tumor length $\geq 4.80 \mathrm{~cm}$, non-uniform scanning density, and arterial phase CT value lower than 72.6 hu indicate high-risk SBGISTs.

\section{Declarations}

\section{Availability of data and materials}

The data sets analyzed during the current study are available from the corresponding author on reasonable request.

\section{Acknowledgements}

Not applicable. 


\section{Funding}

Research reported in this publication was supported by the Fujian natural fund project under Award Number 2018J01249. The content is solely the responsibility of the authors and does not necessarily represent the official views of the Fujian natural fund project. Fujian natural fund project had no role in the study design; the collection, analysis and interpretation of data; the writing of the report; or the decision to submit the article for publication.

\section{Author information}

Affiliations

Provincial Clinical College, Fujian Medical University, Department of Radiology, Fujian Provincial Hospital, Fujian Fuzhou 350001

Lan Yanfen, Chen Shaobin, Ma Mingping

Provincial Clinical College, Fujian Medical University, Department of Critical Care Medicine, Fujian Provincial Hospital, Fujian Fuzhou 350001

Chen Lixun

\section{Ethics approval and consent to participate}

The study was conducted in accordance with the Declaration of Helsinki, and the protocol was approved by the Ethics Committee of Fujian Provincial Hospital ه2020QN0101区. All subjects gave their informed consent for inclusion before they participated in the study.

\section{Consent for publication}

Not applicable.

\section{Competing interests}

Declare that the authors have no competing interests as defined by BMC, or other interests that might be perceived to influence the results and/or discussion reported in this paper.

\section{Authors' Contributions}

Yanfen Lan and Lixun Chen wrote the main manuscript text and Shaobin Chen,Mingping Ma prepared figures 1-2. All authors reviewed the manuscript.

\section{Corresponding author}

Ma Mingping

\section{References}

1. Vijay P Agrawal NW, al e. Perforation of gastric gastrointestinal stromal tumor A rare phenomenon \& review of literature. International Journal of Biomedical And Advance Reasearch. 2014. 5(10): 485-487.

2. Attaallah W, Coşkun Ş, Özden G, et a. Spontaneous rupture of extraluminal jejunal gastrointestinal stromal tumor causing acute abdomen and hemoperitoneum. Ulus Cerrahi Derg. 2015. 31(2): 99-101.

3. Yang Z, Wang F, Liu S, et a. Comparative clinical features and short-term outcomes of gastric and small intestinal gastrointestinal stromal tumours: a retrospective study. Sci Rep. 2019. 9(1): 10033.

4. Li Yongsheng CD, Jiang Han ea. Diagnosis and treatment of duodenal gastrointestinal stromal tumors. Chinese Journal of Digestive Surgery. 2014. 13(10): 793-796.

5. Chinese Society of Gastrointestinal Surgery CSoSMA, al e. Chinese expert consensus on whole-process management of gastrointestinal stromal tumor (2020 edition). Chinese Journal of Practical Surgery. 2020. 40(10): 1109-1119.

6. Demetri GD, von Mehren M, Antonescu CR, et al. NCCN Task Force report: update on the management of patients with gastrointestinal stromal tumors. J Natl Compr Canc Netw. 2010. 8 Suppl 2(0 2): S1-41; quiz S42-4.

7. consensus 2Cegogstp. Expert consensus on diagnosis and treatment of gastrointestinal stromal tumors in China (2017 Edition)Pathological interpretation. Chinese Journal of Pathology. 2018. 47(1): 2-6.

8. Kosemehmetoglu K, Kaygusuz G, Fritchie K, et al. Clinical and pathological characteristics of gastrointestinal stromal tumor (GIST) metastatic to bone. Virchows Arch. 2017. 471(1): 77-90. 
9. Alok KumarTiwari AKC, al e. Primary Extra-Gastrointestinal Stromal Tumor (GIST) arising from mesentery of small bowel and presenting as abdominal mass. Open Journal of Gastroenterology. 2013. 3: 267-271.

10. Baheti $A D$, Shinagare $A B, 0 \& \# 39$, et al. MDCT and clinicopathological features of small bowel gastrointestinal stromal tumours in 102 patients: a single institute experience. Br J Radiol. 2015. 88(1053): 20150085.

11. Mami Yamamoto KY, al e. Obscure Gastrointestinal Bleeding Due to a Small Intestinal Gastrointestinal Stromal Tumor in a Young Adult. Case Rep Gastroenterol. 2016. 10(3): 668-673

12. Wan $\mathrm{W}$, Xiong Z, Zeng $\mathrm{X}$, et al. The prognostic value of gastrointestinal bleeding in gastrointestinal stromal tumor: A propensity score matching analysis. Cancer Med. 2019. 8(9): 4149-4158.

13. Oncology ECogstoCsoC. Chinese consensus on diagnosis and treatment of gastrointestinal stromal tumors (2017 Edition). Chinese Journal of Cancer Research.. 2018. 4(1): 31-43.

14. Liu X, Qiu H, Zhang P, et al. Prognostic factors of primary gastrointestinal stromal tumors: a cohort study based on high-volume centers. Chin J Cancer Res. 2018. 30(1): 61-71.

15. Patil DT, Ma S, Konishi M, et al. Utility of BRAF V600E mutation-specific immunohistochemistry in detecting BRAF V600E-mutated gastrointestinal stromal tumors. Am J Clin Pathol. 2015. 144(5): 782-789.

16. Chen Z, Yang J, Sun J, Wang P. Gastric gastrointestinal stromal tumours $(2-5 \mathrm{~cm})$ : Correlation of CT features with malignancy and differential diagnosis. Eur J Radiol. 2020. 123: 108783.

17. Huh CW, Jung DH, Kim JS, Shin YR, Choi SH, Kim BW. CT Versus Endoscopic Ultrasound for Differentiating Small (2-5 cm) Gastrointestinal Stromal Tumors From Leiomyomas. AJR Am J Roentgenol. 2019. 213(3): 586-591.

18. Zhou C, Duan X, Zhang X, Hu H, Wang D, Shen J. Predictive features of CT for risk stratifications in patients with primary gastrointestinal stromal tumour. Eur Radiol. 2016. 26(9): 3086-3093.

19. Chen T, Xu L, Dong X, et al. The roles of CT and EUS in the preoperative evaluation of gastric gastrointestinal stromal tumors larger than $2 \mathrm{~cm}$. Eur Radiol. 2019. 29(5): 2481-2489.

20. Mazzei MA, Cioffi Squitieri N, Vindigni C, et al. Gastrointestinal stromal tumors (GIST): a proposal of a "CT-based predictive model of Miettinen index" in predicting the risk of malignancy. Abdom Radiol (NY). 2020. 45(10): 2989-2996.

21. Xia Guojin HZ, He Yulin ea. The correlation between MSCT enhanced findings and pathologic risk in the patients with primary small intestinal stromal tumor. Journal of Practical Radiology. 2017. 33(6): 64-68.

22. Feng F, Wang F, Wang Q, et al. Clinicopathological Features and Prognosis of Gastrointestinal Stromal Tumor Located in the Jejunum and lleum. Dig Surg. 2018: 1-5.

23. Su Q, Wang Q, Zhang H, et al. Computed tomography findings of small bowel gastrointestinal stromal tumors with different histologic risks of progression. Abdom Radiol (NY). 2018. 43(10): 2651-2658.

24. Feng C, Lu F, Shen Y, et al. Tumor heterogeneity in gastrointestinal stromal tumors of the small bowel: volumetric CT texture analysis as a potential biomarker for risk stratification. Cancer Imaging. 2018. 18(1): 46.

\section{Figures}
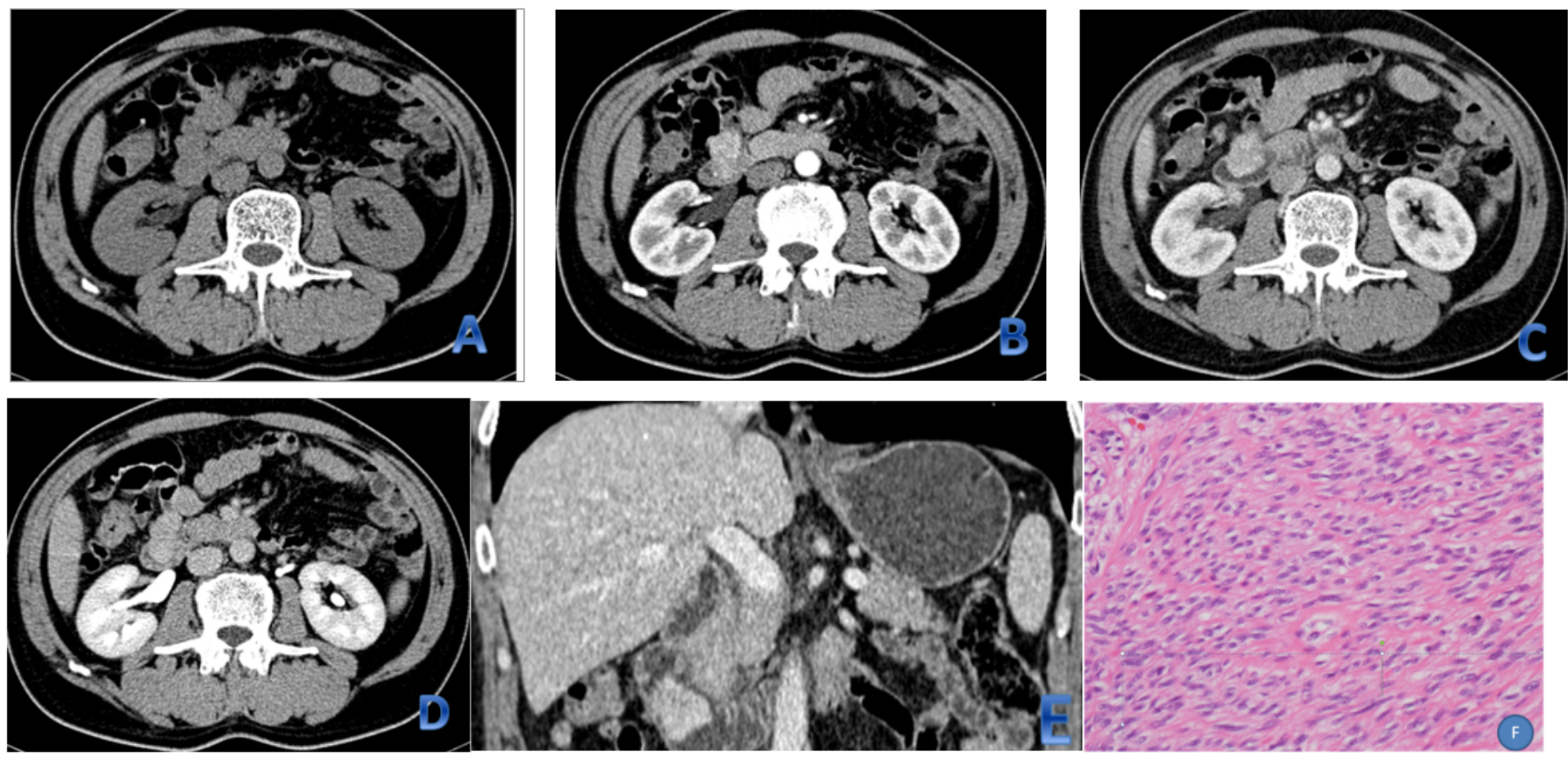

Page $8 / 10$ 
Figure 1

MSCT scanning and reconstruction images and pathological results of duodenal gastrointestinal stromal tumors MSCT scan showed slightly lower and equal density in transverse section (A), obvious enhancement in arterial phase (B), continuous enhancement in venous phase (C), and decreased enhancement in delayed scanning (D). In coronal position (E), the lesion was located in duodenal cavity, with clear boundary and uniform density. The pathological risk grade was low (F).
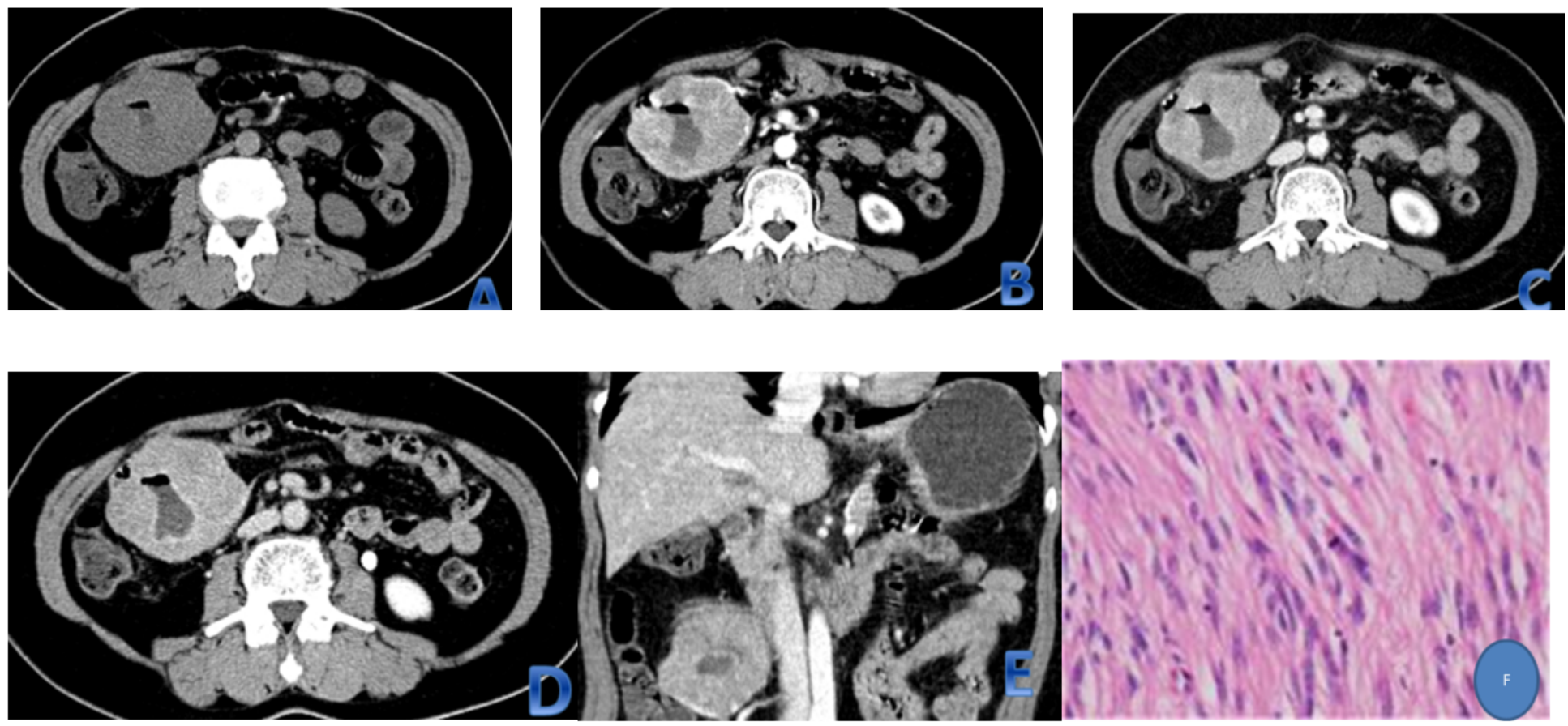

Figure 2

four phase MSCT scanning and reconstruction images and pathological results of ileal gastrointestinal stromal tumors MSCT showed a slightly lower density in the horizontal section (A) of plain scan, and patchy necrotic areas were seen in it. In the arterial phase (B) of enhanced scan, the lesions showed uneven and obvious enhancement, with multiple small vessel branches supplying blood in it, no enhancement in the necrotic cystic area, slightly weakened enhancement in venous phase (C) and delayed phase (D), and mesenteric artery branches supplying blood in coronary position (E), The pathological risk level was high (F).

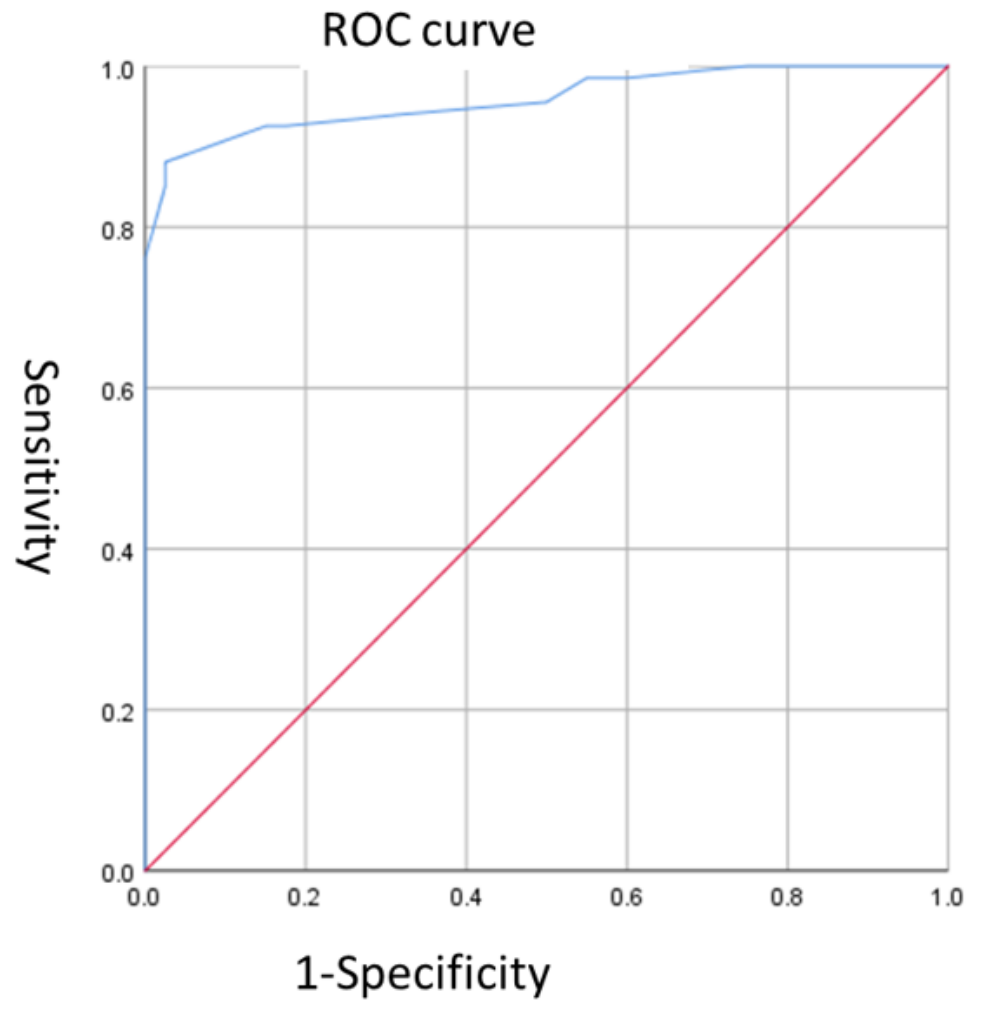


Figure 3

ROC curve analysis of lesion length diameter in the higher risk group

\section{ROC curve}

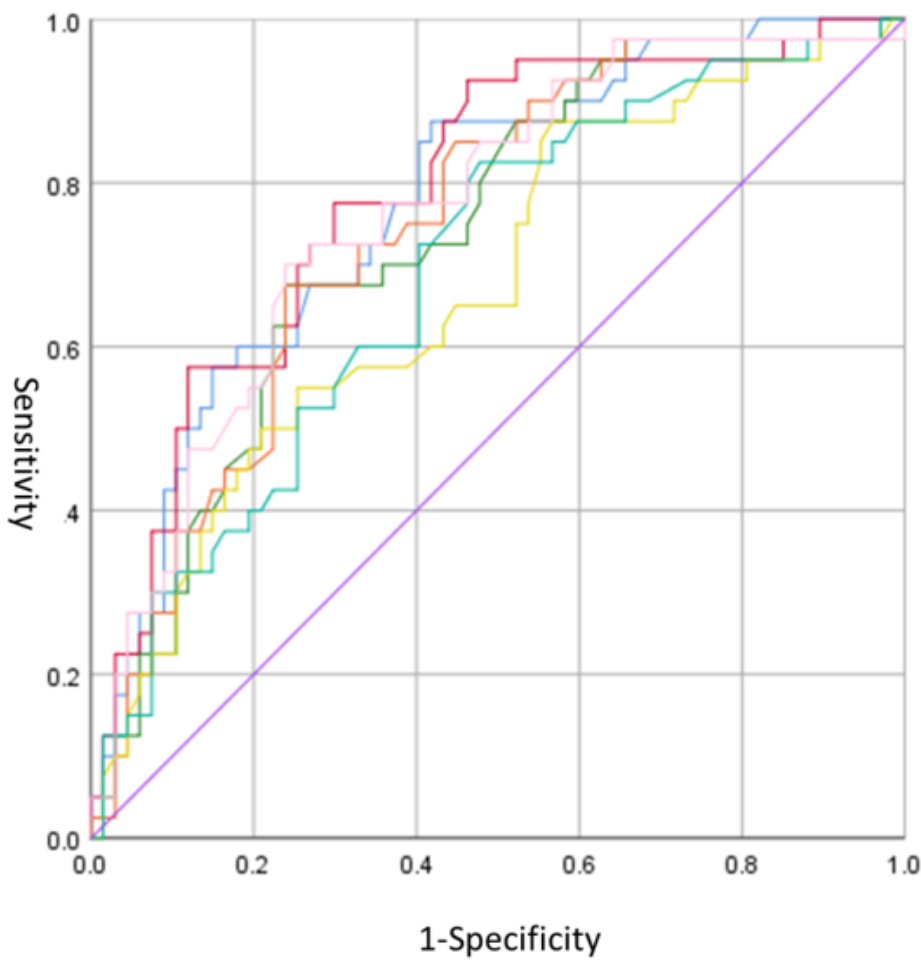

Sources of curves

_ Arterial phase
Arterial phase increment
- Venous phase
- Venous phase increment
Delayed phase
Delayed phase increment
Enhanced net
Reference lines

Figure 4

ROC curve analysis of CT findings on contrast-enhanced scans in the lower risk group 